\title{
DirecionaSUS: Mobile Applications and Stochastic Timed Petri Nets for Referral of Patients and Dimensioning Hospital Beds for Health Services
}

\begin{abstract}
Paulo Rodrigues de Souza, Diego Rodrigues Andrade Simão, Alice Ramalho Tavares, Anielle Rodrigues da Silva, Anna Zarife Feres Micheletti, Anne Lara Moreira Gomes, Danielle Ruas Santos, Eduarda Luiza Loschi de Araújo, Fernanda Vasconcelos Pacheco, Gabriela Raposo Tavares, Stéphannie Freitas Miranda e Souza, Luiz Melk de Carvalho, Diva de Souza e Silva Rodrigues, Flávio Henrique Batista de Souza
\end{abstract}

Centro Univesitário de Belo Horizonte - UNIBH, Brazil

\begin{abstract}
.
The dimensioning of health services is one of the greatest challenges in the city of Belo Horizonte, especially in times of crisis, such as COVID-19. This research demonstrates a solution that integrates mobile tools, cloud computing and stochastic timed Petri nets to optimize the availability of hospital beds. Firstly, a mobile solution was developed, named DirecionaSUS®, capable of directing the patient to the nearest care unit, with the appropriate medical specialty. The guidance is performed after the patient inserts the symptoms that he is having in the application. The application is connected to a second solution: a data analytics developed in cloud computing, capable of demonstrating information about the main demands, with averages, heat maps of the concentrations of requests and other functionalities. The two tools serve as a reference for a last one: a simulator in stochastic timed Petri nets, capable of simulating hospitals bed demands, considering the times of assistance and the definitions of the Manchester protocol. Experiments with data and real hospitals were carried out and the ability of intelligent bed allocation management in hospitals was demonstrated.
\end{abstract}

Keywords: Stochastic Timed Petri Nets, Health Services Dimensioning, Cloud Computing, Mobile Applications, Hospital Beds Demands.

\section{Introduction}

Health promotion and protection are essential for human well-being and for sustainable economic and social development. Because it is the greatest good of a human being, it is of paramount importance to manage organizations that help maintain human life efficiently, given that people in most countries classify health as one of the highest priorities, surpassed only by economic concerns (Spencer et al, 2019). Health services have been seeking to improve their processes in order to offer more favorable environments for the reception of patients. Common problems in this environment such as: delay in making appointments, exams, surgeries and other procedures, overcrowding, lack of hospital supplies, poor 
management of resources, among others, are some difficulties presented in the area under study (Pohjosenperä et al., 2019).

A health system is composed of several functional parts, among which are: infrastructure, organization, management, financing and provision of care. Currently, Brazil is going through a turbulent situation where hospital management faces several difficulties (scarcity of financial resources, poor quality of care, huge waiting lines, among others.). With an extremely important role in this scenario emerges the Emergency Services, where the time and waiting time is a determining factor for the success of the service provided by the supplier agency. In addition, an aggravating factor: in 2020, with the pandemic process due to COVID-19, the health scenario in Brazil was seriously affected with a lack of resources, guidelines and the need for strategic tools for managing care beds (Cimerman et al., 2020).

This paper focuses on a medical care management problem, focusing on a proposal to support SUS, the Unified Health System of Brazil. According to Giovanella et al. (2019) the SUS care system is a model based on the hierarchy of health actions and services by levels of complexity. The purpose of this system is that cases of less urgency can be resolved in instances that do not reach specialized centers of high complexity, improving the efficiency and effectiveness of the entire system. Many emergency care services live in long lines where people dispute the service without any criteria other than the time of arrival. The nondistinction of risks or degrees of suffering causes some cases to get worse in the queue, sometimes occurring the death of people due to non-attendance at the appropriate time (Ministério da Saúde, 2009).

The work presented aims to demonstrate a possible optimization of care management in hospitals and health posts in a strategic way using Data Analysis. Thus, the general objective of this work is to present a structured application consisting of the aforementioned technologies, to demonstrate the viability of a tool capable of strategically managing the demand of SUS-MG service users, according to their needs. As specific objectives the work aims to use and optimize the structure based on the mobile application generated for Dir Dira SUS; develop the data capture process via Cloud Computing in order to create a database with relevant data for use in the strategic medical management of SUS-MG; according to the data generated and collected, create a structure that, through a Data Analysis process, is able to strategically manage the medical demands of SUS-MG users; and finally, to present an experiment with data collection and evaluation from a care unit, in order to simulate bed demands upon the arrival of patients through the Sthocastic Timed Petri Nets (STPN).

Through a mobile application it will be possible to collect and generate the information that will be stored in Cloud Computing technology, which has the capacity to distribute computing services in a flexible way, representing a more efficient use of resources, saving costs and time. Data analysis, which is the process of systematically applying statistical and / or logical techniques to describe and illustrate, condense and recapitulate and evaluate data, using computational tools, in order to find information that is relevant in decision making. With them, it is possible to guide simulations oriented to discrete events, with tools such as STPN. With the arrival times of the patients, it is possible to simulate the demand and concentration of the queues, and to evaluate the efficiency of the number of available beds, considering the Manchester protocol (Safonov, 2016; Washington et al., 2020; Lisboa et al., 2019). 
This work is justified by the huge volume of queues at hospitals and health posts that use the SUS service. There are already solutions proposed by the government, such as DigiSUS (recently launched in 2019), now called ConecteSUS, to assist patients (Minitério da Saúde, 2019). However, as a contribution, this paper demonstrates not only the mobile solution, but a four-pronged study (mobile solutions, cloud computing, data analysis and modeling and simulation) that ranges from guiding the patient to assessing the demand for beds. Another gain of the research is that, with the use of DirecionaSUS, the data could, with the permission of the government, be made available to private or public institutions, be able to propose, with their own tools, solutions and support to the Ministry of Health, promoting research with real databases, without risks with sensitive data.

\section{Methods}

The methodology developed in this work is based on experimentation and analysis in two stages: the development of a mobile tool, with support for cloud computing and data analysis, to assist patients and an evaluation, with real data, provided by a unit of service, for experimentation and simulation of bed rows. The intention is that with the use of the mobile solution, a cloud database is fed so that it can: guide a data analysis tool and coordinate a simulation tool focused on bed waiting time.

\subsection{Implementation of the patient guidance structure and data consolidation}

During the work development process, it was firstly sought to carry out the prototyping and optimization of the app used for the generation of information. Then it was proposed to develop a data consolidation architecture based on cloud computing. The data is collected during the app usage, where it will be stored in a cloud solution so that data analysis and experimentation can be carried out. Thus, an analysis was carried out to obtain information that may be relevant for strategic management in the field of medicine, focused on service provision.

The concept of Data Analysis was used to assist in the recommendation of mobile units and management of health posts through the analyzed information, where a classification will be made on which region needs help according to the volume of patients directed to each region, also considering the date, time and risk class assigned to each patient. Thus, the solution named DirecionaSUS was developed.

\subsection{Experiments with real data for bed demand simulation}

With the mobile solution defined, one of the desired feasibility is that a simulator is able to understand and evaluate the bottlenecks of bed demands following the Manchester protocol. Thus, in parallel with the development of the mobile solution, a survey was carried out in a hospital, with real demand data. The hospital under study is located in the metropolitan region of Belo Horizonte (a Brazilian city) and serves an average of 150 patients per day according to information provided by the staff. With the level of complexity of the activities provided as medium complexity, that is, formed by specialized services on an outpatient and hospital level, with intermediate technological density. It has 31 beds, with the help of a team of 85 doctors. 
The Manchester Protocol is used, with the purpose of prioritizing urgent and emergency care. In this Protocol, patients are treated based on the risk potential and not on the order of arrival. Another considerable piece of information regarding hospital management is that the Ministry of Health monitors two key indicators to assess hospital institutions: occupancy rate and permanence rate. Results considered positive are those that include low stay rates and high occupancy rates. In order to calculate the number of patients to be sampled, data were collected on the databases provided by the institution. It was a very slow process and presented several obstacles. In a first definition, only the collection, without a treatment of the data representation, was proposed. However, during the analysis process, it was necessary to validate and filter the collected data.

For the computational model, the required information was defined as: Rate of arrival and flow of patients, distribution of patients according to criticality, duration of various activities involved, amount of resources involved. The development was based on the main problem, the long waiting time in hospitals, with the main objective of reducing this waiting period using STPNs. It is necessary to feed the model with the arrival rates of the patient flow, understanding the care processes (taking into account care priorities or not), survey of available resources and their arrangement in the existing layout in the environment. The entire model generates as output characteristics of specific system operation that is crucial to identify the bottlenecks in the process.

\section{Results}

\subsection{Patient support structure and data consolidation}

\subsubsection{Mobile Implementation}

A mobile solution was developed that acts on the process of providing information and generating routes, for the Health Services of Belo Horizonte. For the construction of the work and development of the application, it was necessary to identify which agents would be involved in the process. Two agents were identified, with the Patient as the requesting agent and the SUS as the service provider agent. When using the application, the patient has the option of viewing information from health services as well as requesting the generation of the route through the reported symptoms. The route is generated through automatic guidance, based on the patient's distance to the nearest service, or by the patient's choice. In Figure 1, the sequence of activities necessary to serve the user is available, which must be performed by the application. 


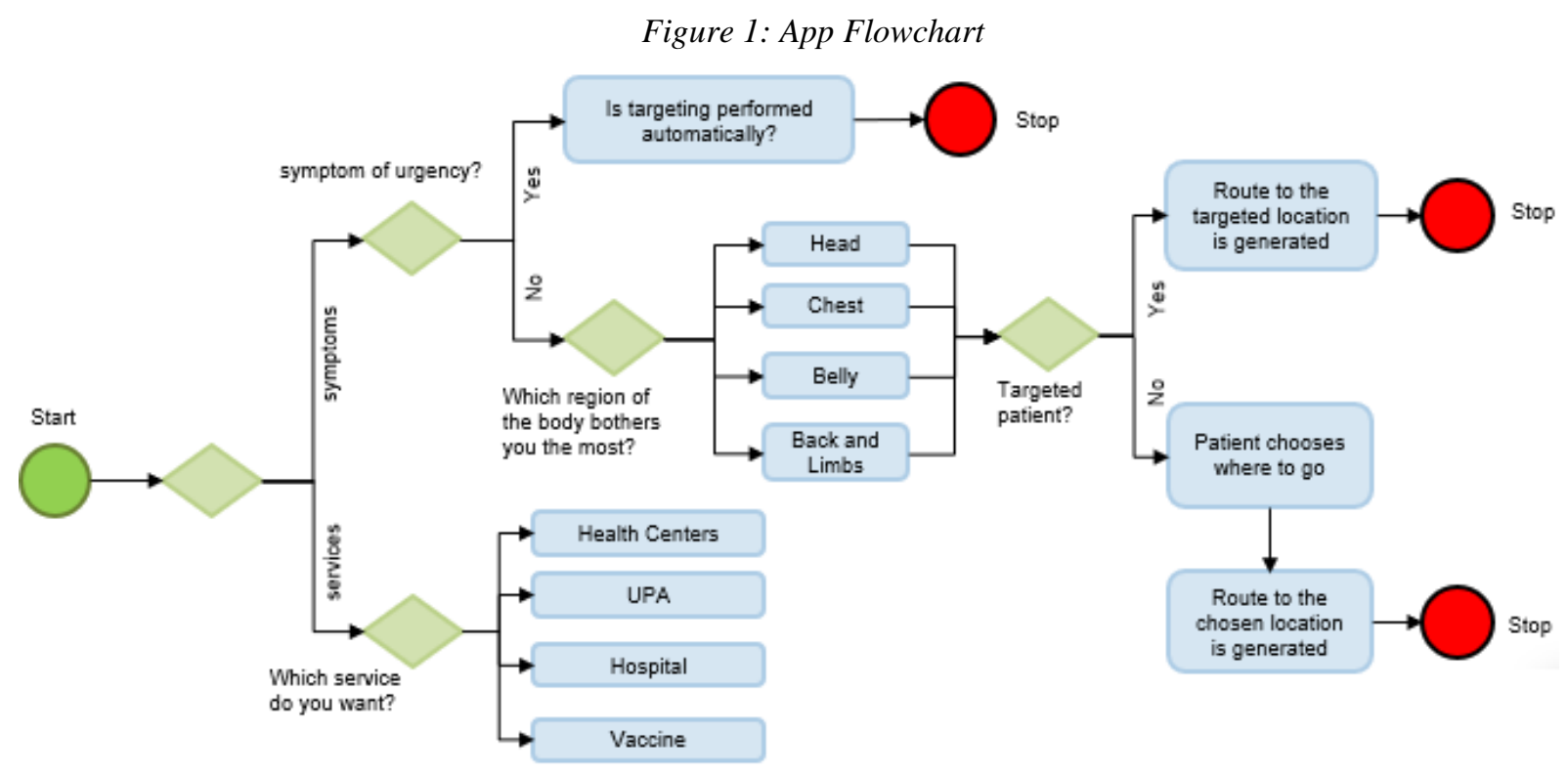

Source: (Authors)

In this case, the hierarchy made available obeys the sequence of data collected / displayed by the solution. The app has as its initial screen an interface that allows the user to login into the application through his email and a password previously registered. If the patient still does not have a record, on the same screen the individual has the option of being able to perform his record, being directed to a new page, where they must fill in his data. The patient must register using their name, a valid email and password. After filling in, the user must save this information and a message will appear in the center of the screen, informing that the registration will be completed after checking the account through a link sent to the registered email.

Upon logging in, the patient is directed to the main menu of the application. The patient will have access to the options of "Services", "Symptoms", "Profile" and "Meet the SUS", where they can view information related to Health Services, learn more information about the Unified Health System, as well as inform the region and the symptoms they are feeling, so that the direction is done and the route is generated. From the generated route, when the patient clicks on the destination marker, a set of three questions is presented, where the patient signals if he has already undergone the reception, then indicates what were their risk rating and finally, if they have already been attended.

The application was developed using the Java programming language, the Android Studio IDE and the Firebase cloud solution for data storage. The structure of the application consists of the communication between the app and the database for storing and searching data and the communication of the application with the OSMdroid map API. Thus, the communication flow of the application between the technologies can be viewed through Figure 2. Thus, in Appendix I, the interfaces developed are presented. 
Figure 2: Mobile and Cloud Computing Structure

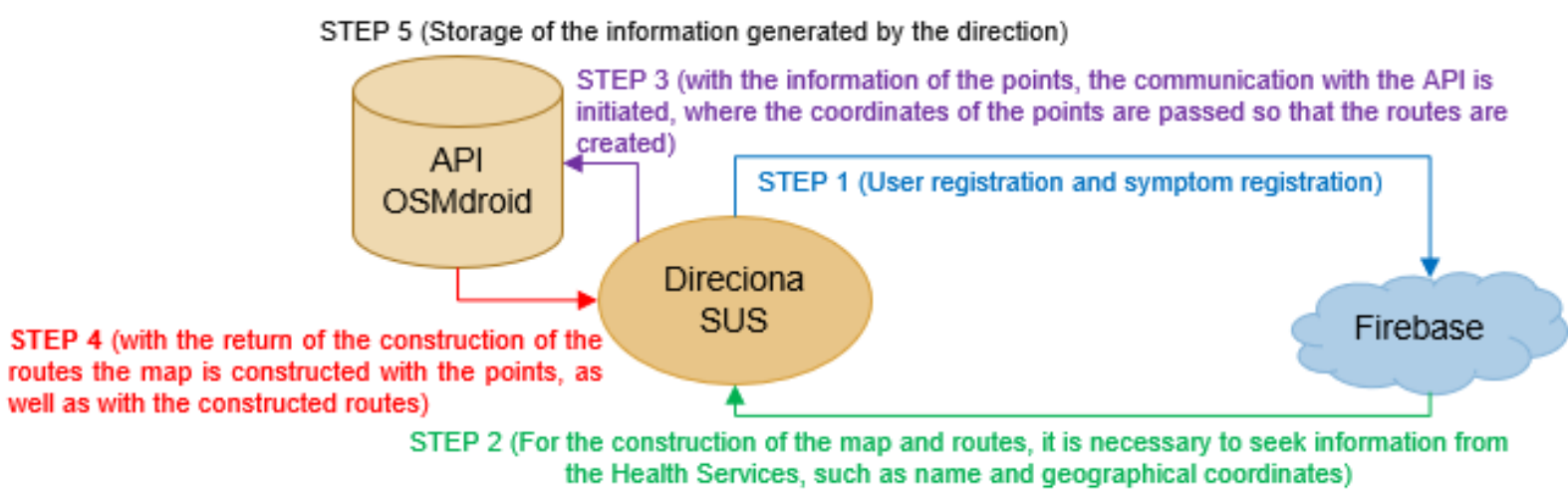

Source: (Authors)

\subsubsection{Data Analysis Tool}

For data analysis, a mapping tool was used during the development to generate the heat map on demand, which is compatible with Javascritp, Python and R. It was chosen to use the development option by Javascritp, because it is best suited to the scope of the project. Such a tool has the purpose of making analyzes to the public service manager feasible, in order to generate service allocation strategies, according to the concentration of demands.

As the data is available in the Cloud, other institutions, previously registered and licensed by SUS, could use such data in the cloud for studies. The proposed, but not unique, approach is to make a geographic assessment using heat maps. This proposal for the first exploration of the data collected by using DirecionaSUS aims to enable the user to see concentrations of demand in Belo Horizonte, as well as the average time of service in each unit and possible cases of epidemic diseases. The heat maps will interact with the maps of the city of Belo Horizonte (which can be expanded to other cities).

Heat maps are generated according to the service demands that users present when using the app. For the construction of the heat map, the following data were used: Health Services Coordinates; Name; Symptom region; Risk rating; Targeting time; Targeting date. A total of 124 targeting samples were randomly generated. The data presented are fictitious but based on situations that can be obtained in reality. On the map it is possible to filter the information presented, by Attendance Services, body region, date, time with all the information together.

Figure 3 shows a consolidation of the requests made. As the filter requested all points, there was no limiting element. All service consumption points were analyzed, where the intensity of the "heat" provided by the map only considers the number of service demands, without distinguishing, for example, whether they are Hospitals or PSUs, or the region of the registered symptoms. It can be considered the most widespread and least objective of the options. 
Figure 3: Data Analysis Tool Developed (Evaluation via heat map)

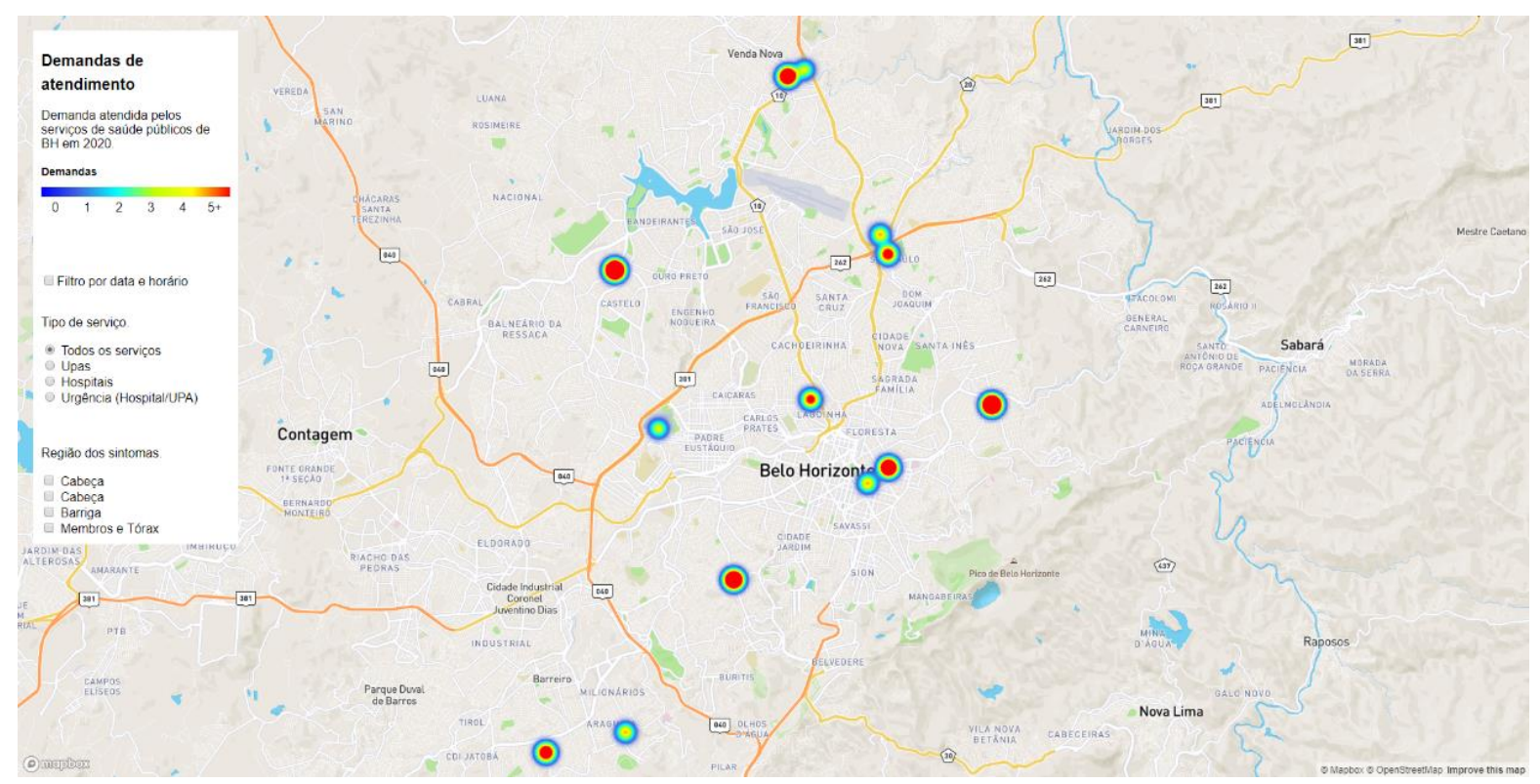

Source: (Authors)

\subsection{2 Experiments with real data to assess bed demand}

In this step, fundamental data will be presented for the development of the work, where a collection was made in order to provide information to add meaning to the results that is generated.

\subsubsection{Data Collection}

The data obtained were provided by the Hospital voluntarily for analysis through reports from the internal Screening System. Two types of reports, of different formats and periods, were analyzed, considering that the Institution underwent a software exchange during the current collection year. The first report provided covered the first half of 2017 and the second from the new system with information from July 1, 2017 to September 1 (this one with much more coherent information).

There were several challenges in the data collection process for the development of the work, such as: conflicting data, screening record system showing failures when exporting reports for analysis, typos for classification of information, lack of standard in saved information, important fields for analysis of blank data, among others. A significant amount of data that had to be disregarded due to errors and/or inconsistencies and this was necessary to obtain a more precise analysis (figure 4).

Some care was taken in order to respect the particularities of the data provided, such as, for example, maintaining the confidentiality of some confidential data. In view of the large volume of activities present in the context of work experienced by employees, the high demand for highly complex tasks is notorious for solving them very quickly. Among the professionals who work in the process, some have volunteered (on some occasions) to answer questions about the hospital's operating process. For this reason, data collection was carried out with the least possible intrusion. It is recognized that the difficulties faced characterize factors that impact on the studied sample directly or indirectly (in addition to the fact that 
database 1 accounted for only approximately $72 \%$ of the data provided and database 2 with only $32 \%)$.

Figure 4: Filtering process (database 1 and 2)

\begin{tabular}{|c|c|c|c|}
\hline \multicolumn{2}{|c|}{ Filtering correct records - database 1} & \multicolumn{2}{|c|}{ Filtering correct records - database } \\
\hline initial database & 19311 & initial database & 9190 \\
\hline No date recording & 18639 & negative time & 9183 \\
\hline No time recording & 17967 & service stop time null & 9166 \\
\hline No flowchart described & 15982 & service start time null & 9009 \\
\hline database useful & 15007 & database useful & 29 \\
\hline
\end{tabular}

Source: (Authors)

\subsubsection{Service Flow}

In order to better understand the process of patient arrival at the Emergency Room, four interviews were conducted with a professional from the organization, responsible for managing part of the hospital, thus generating a flowchart of care covering the various possibilities that a patient may encounter when being attended to.

The construction of this flow took a period of three months, in view of its complexity. Thus, this flowchart is considered a relevant contribution to the institution, given that there was nothing similar before the study was carried out as presented. In addition to the contribution of the delimitation and understanding of the process, the analysis of the flow of activities guides the analysis of a customer's arrival time (shown in Figure 5a) and screening time (shown in Figure 5b), which are extremely relevant information, such as will be described below, during the analysis via Petri nets. The flow of care is based on the principle of common care through receptionists and nurses who refer patients for care according to the classification proposed by the Manchester Protocol and according to the specialty required by the symptoms observed.

Using data from the first semester available in database 1, it was possible to identify various informations. It was observed in Figure $5 \mathrm{c}$ what is daily reported by hospital employees, $83 \%$ of all consultations performed are classified with the color green. This classification is intended for care with low severity, which underlies the care of these cases through the fast track system, which is oriented towards more care. This system aims to optimize care through a body of doctors designed to provide care directed to low-risk symptoms. The services classified as red are those with the most complex situation and have the highest priority in the service queue. As mentioned during the study, the hospital meets procedures of medium complexity, so cases that fit this classification are only stabilized and then transferred to hospitals that have resources for the treatment of cases with high complexity. 
Figure 5: Statistical analysis
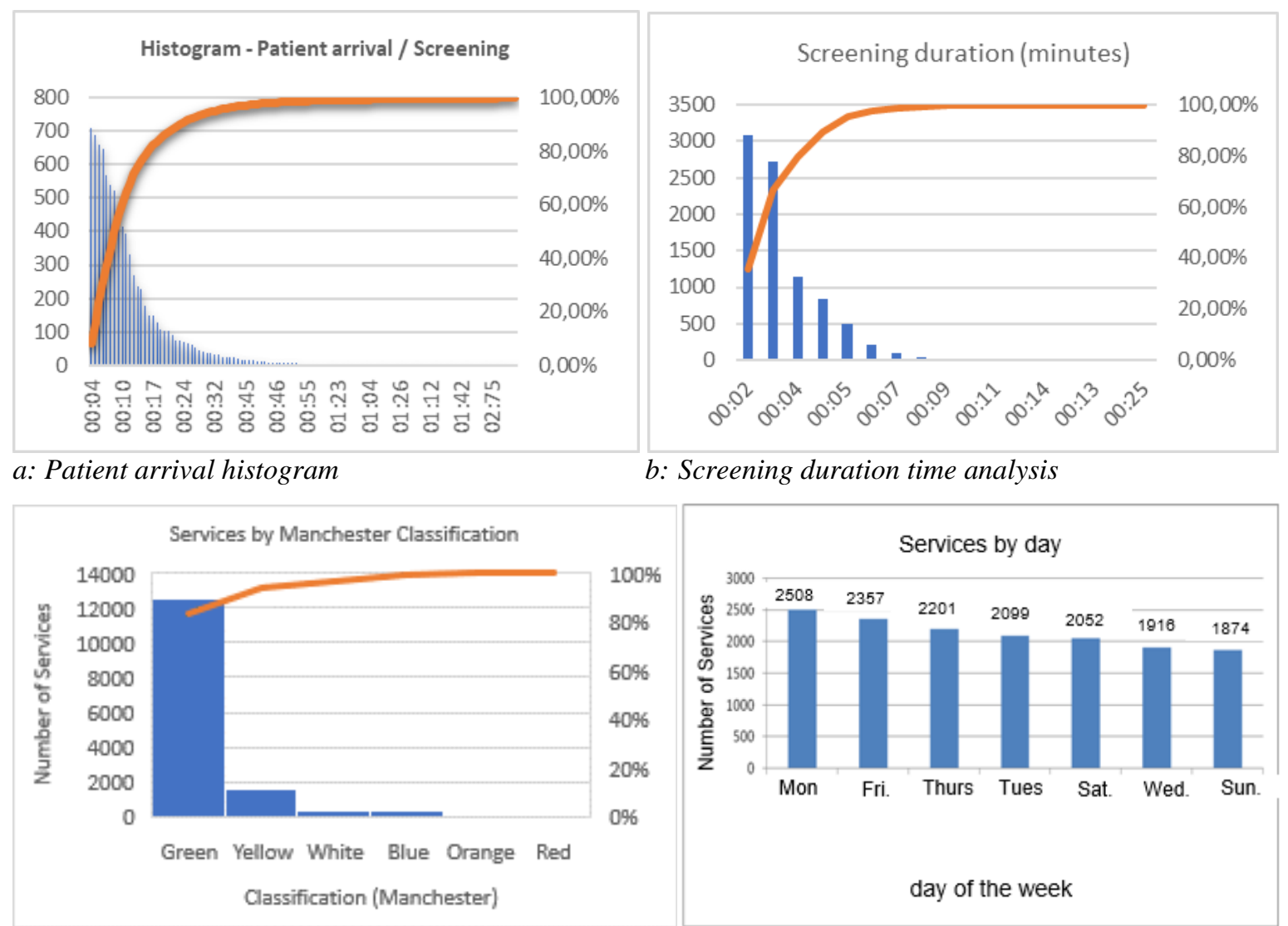

c: Attendance concentration by Manchester classification d: Services per day of the week Source: (Authors)

Of all the attendances registered in the first half of 2017 (and according to the data shown in Figure 5c), the type of attendance (according to the Manchester protocol) is very irregularly distributed among the specialties offered by the hospital. It is noteworthy that approximately $83 \%$ are concentrated in the specialty of medical clinic, a fact that is due to the wide general symptomatic care provided by professionals in the clinical area. Also, as evidence found, it is observed that the two days of the week where most of the visits are concentrated are Monday and Friday as shown in Figure 5d.

It was reported by the professionals interviewed at the hospital that this increase in the demand for care is due to the fact that in these two days most of the patients are looking for a medical certificate. Such quantitative surveys had not been carried out, being extremely important for the planning of shifts, planning the consumption of resources, among others.

\subsubsection{Modeling and Simulation via Stochastic Timed Petri Nets}

The Petri Net built (Figure 6) was able to demonstrate the dynamics of hospital care based on data collected from the average time of arrival of patients at the reception and also in the screening. The reception and screening process are carried out by only one professional, with an average arrival rate of 10 minutes, which initially proved to be efficient, considering that the average service time for both processes is 4 and 2 minutes respectively. However, more experiments must be performed, as the sampling behavior is exponentially distributed. 
Figure 6: STPN for assessing patient arrival and waiting time (based on the Manchester protocol)

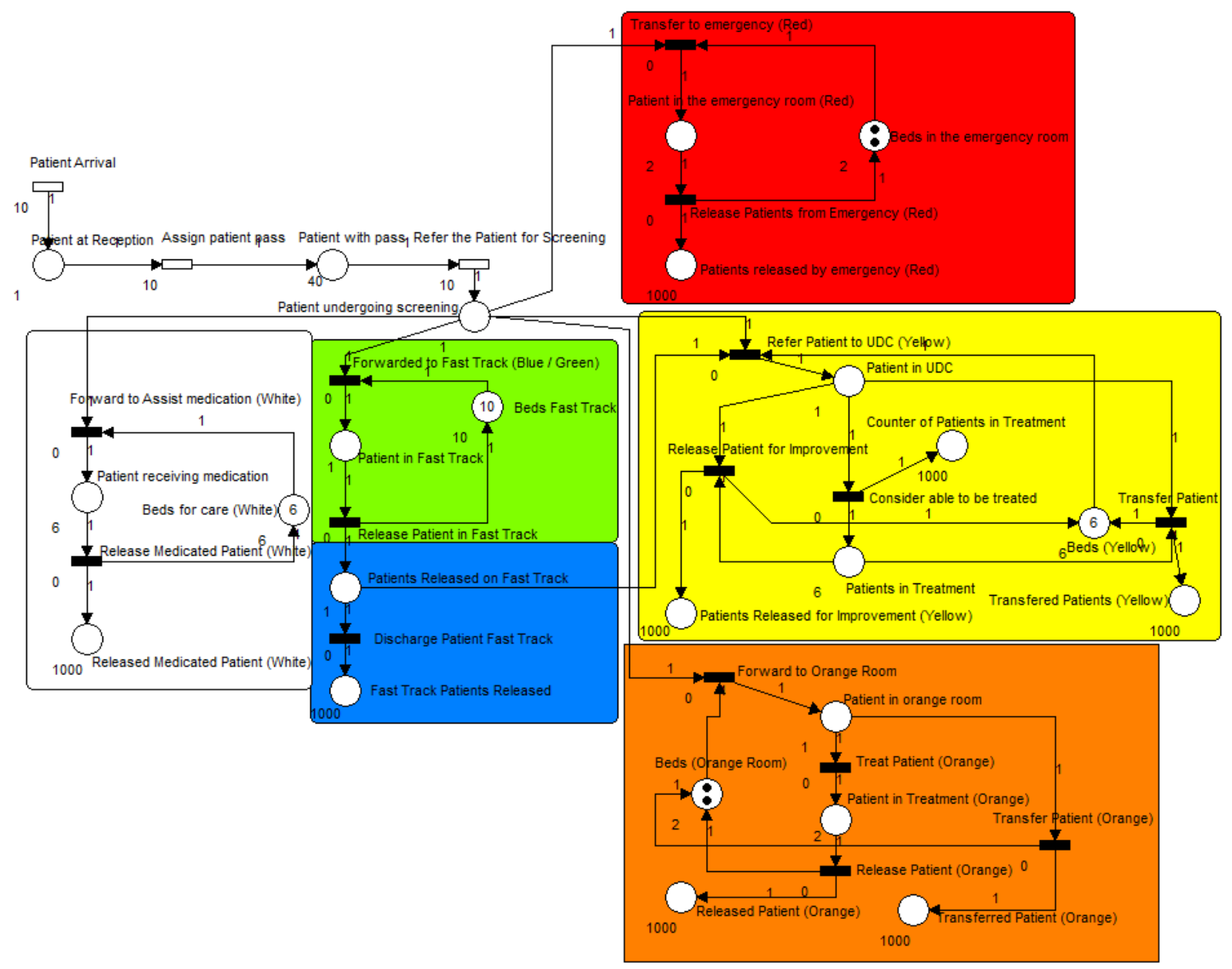

Source: (Authors)

During the experiments, the simulator randomly allocated patients in different classifications, in order to segment the care process. The entire process complied with the Manchester Protocol color bed capacity restriction. However, the work presented limitations during the simulation, having some factors, such as: absence of probabilistic distribution of the patient flow after screening, data recorded manually by employees (which causes noise in the sample), lack of the rate of arrival of care processes etc.

It should be noted that if there is data to feed the model with real times in the transitions, the model will meet, with considerable efficiency, the total demand of the hospital, enabling strategic analyzes guided by scenario simulations. Analyzes, similar to those presented in Figures 5a and 5b, will be necessary for each activity described (in this case, the Petri net, each event represented by a transition).

Even with the difficulties of data collection, through the simulation tool it is possible to verify in hypothetical scenarios, how the beds management in that hospital would be. In an experiment, it was simulated that the referral process through the transitions $T=\{$ Forward to Assist medication (White); Forwarded to Fast Track (Blue/Green) $\}$ had an exponential distribution of mean $\mu=5$ minutes; $T=\{$ Forward to Orange Room; Refer Patient to UDC $\}$ um $=10$ minutes, and $T=\{$ Transfer to emergency $(\operatorname{Red})\} \mu=20$ minutes. For a certain degree of 
realism, the release/transfer time was added to 3 minutes, with times of $\mu=8$ minutes; $\mu=13$ minutes and $\mu=23$ minutes. The result is shown in Figure 7 .

Figure 7: Graphical analysis of bed availability

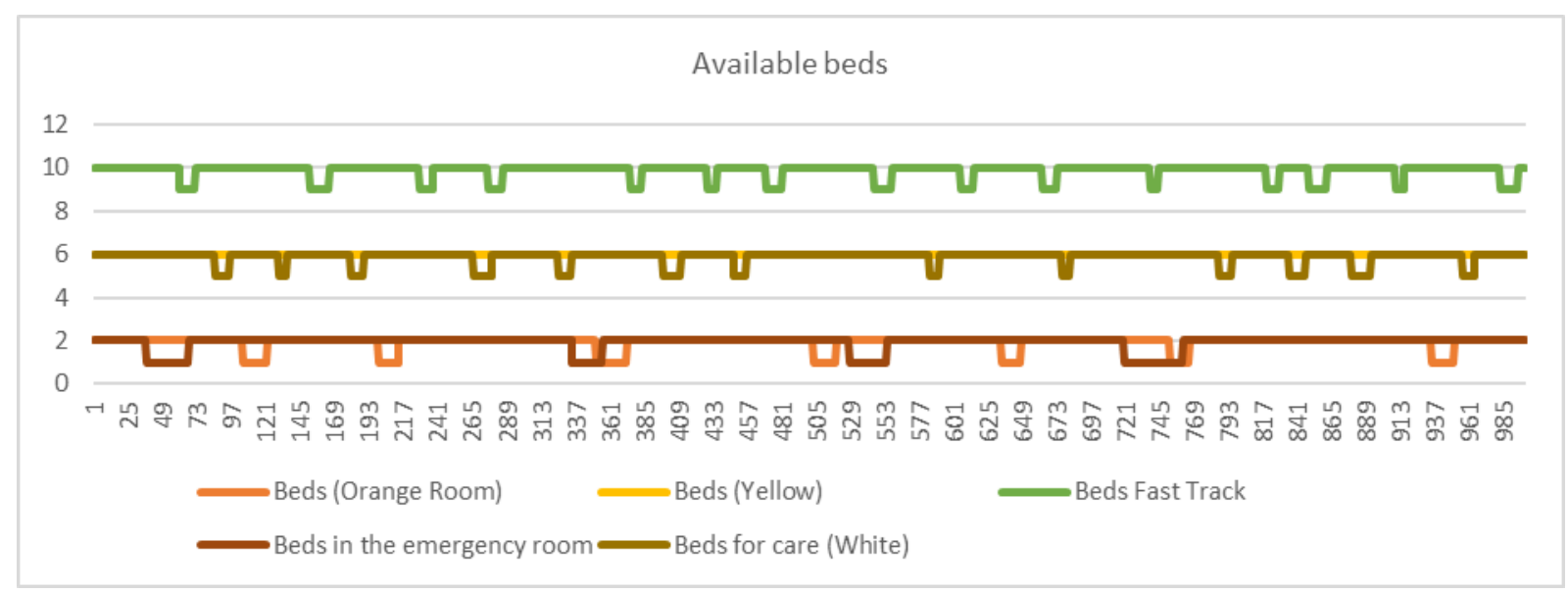

Source: (Authors)

It is suggested that the hospital adopt software that fully registers all possible flows that a patient can acquire within the existing screening system. Require from the software used concise reports that inform, average rate of patient arrival, time of each process etc. It is extremely important that all employees understand the importance of good management based on data and mathematical models for more assertive decisions and, consequently, better patient care.

\section{Conclusion}

The movement of patients to a health service, as well as the large number of queues, is still a problem in the country's health services. The present study aimed to optimize the displacement of patients, as well as to optimize / improve the management of the various Health Services existing nowadays in Belo Horizonte, such as health posts and Hospitals, through the proposal of a data collection and analysis system.

Considering the related works, the DirecionaSUS application also aims to minimize problems in customer service and improve customer service. Through DirecionaSUS a database is generated which, with the use of Data Analysis, it is possible to obtain information that can be used to improve the management of the units as well as in the creation of service strategies.

Based on the results obtained, it was found that it is possible to optimize the displacement of patients as well as to perform better management of Health Services, which would have the main benefit of improving care, reducing the capacity of the places and saving resources. Unlike the services and jobs already available on the market and in the literature, this work not only acts and makes resources available to the user / patient but optimizes and provides resources to service managers. 


\section{References}

[1] Spencer, G., Corbin, J. H., and Miedema, E. (2019). Sustainable development goals for health promotion: a critical frame analysis. Health promotion international, 34(4), 847858.

[2] Pohjosenperä, T., Kekkonen, P., Pekkarinen, S., and Juga, J. (2019). Service modularity in managing healthcare logistics. The International Journal of Logistics Management.

[3] Cimerman, S., Chebabo, A., da Cunha, C. A., \& Rodríguez-Morales, A. J. (2020). Deep impact of COVID-19 in the healthcare of Latin America: the case of Brazil. Braz J Infect Dis.

[4] Safonov, V. O. (2016). Trustworthy cloud computing. John Wiley \& Sons.

[5] Washington, S., Karlaftis, M. G., Mannering, F., and Anastasopoulos, P. (2020). Statistical and econometric methods for transportation data analysis. CRC press.

[6] Lisboa, A. C., De Souza, F. H. B., Ribeiro, C. M., Maia, C. A., Saldanha, R. R., Castro, F. L., \& Vieira, D. A. (2019). On modelling and simulating Open Pit mine through stochastic timed Petri nets. IEEE Access, 7, 112821-112835.

[7] Machado, H. S. V., Melo, E. A., and Paula, L. G. N. D. (2019). Medicina de Família e Comunidade na saúde suplementar do Brasil: implicações para o Sistema Único de Saúde e para os médicos. Cadernos de Saúde Pública, 35, e00068419.

[8] Ministério Da Saúde (2009). Acolhimento e Classificação de Risco nos Serviços de Urgência. Brasília: 2009. Available in http://www.saude.gov.br/

[9] Ministério Da Saúde (2019). Estratégia de Saúde Digital (e-Saúde) para o Brasil: digiSUS. Disponível em: http://www.saude.gov.br/acoes-e-programas/digisus

\section{Appendix I: Interfaces Screenshots}

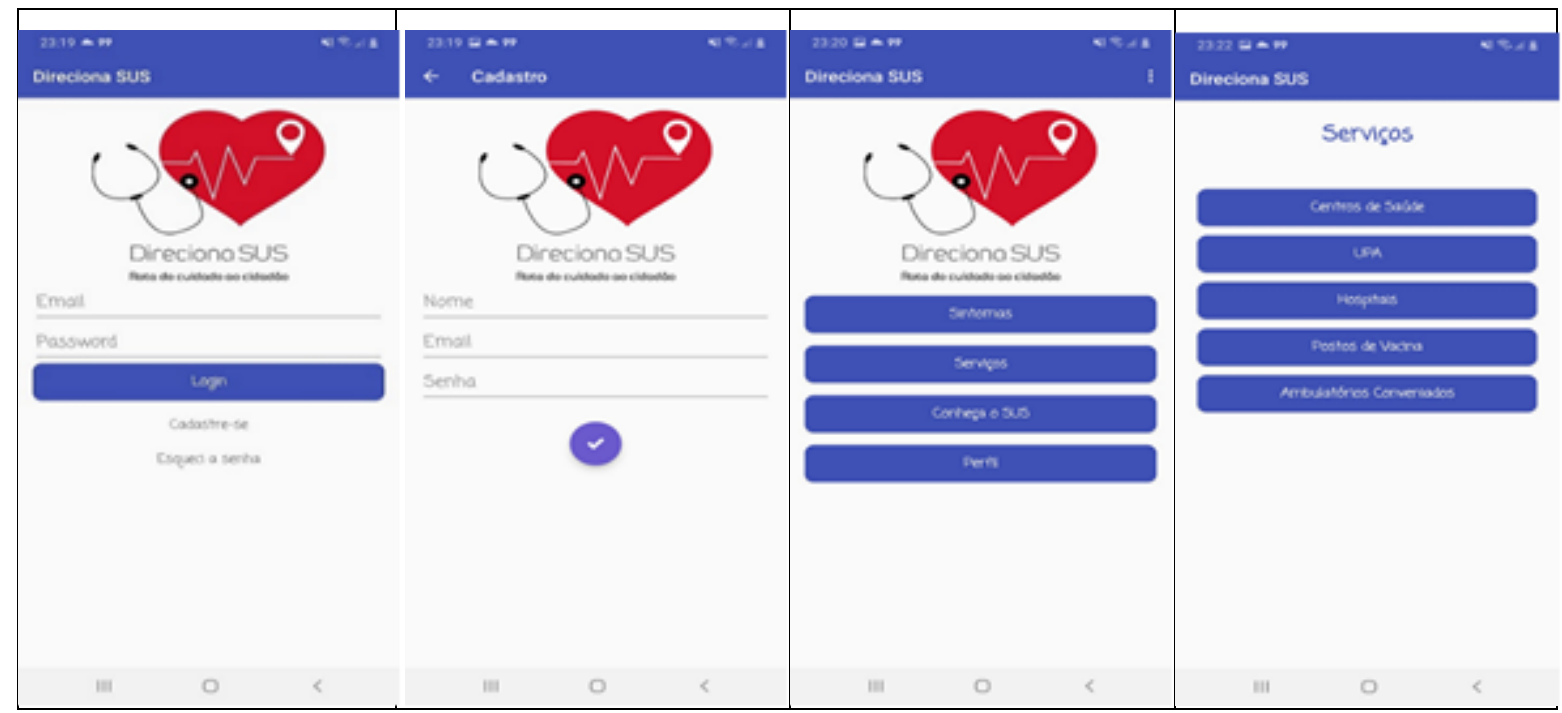


11th International Conference on Research in

ENGINEERING, SCIENCE \& TECHNOLOGY

Budapest, Hungary 19-21 March, 2021

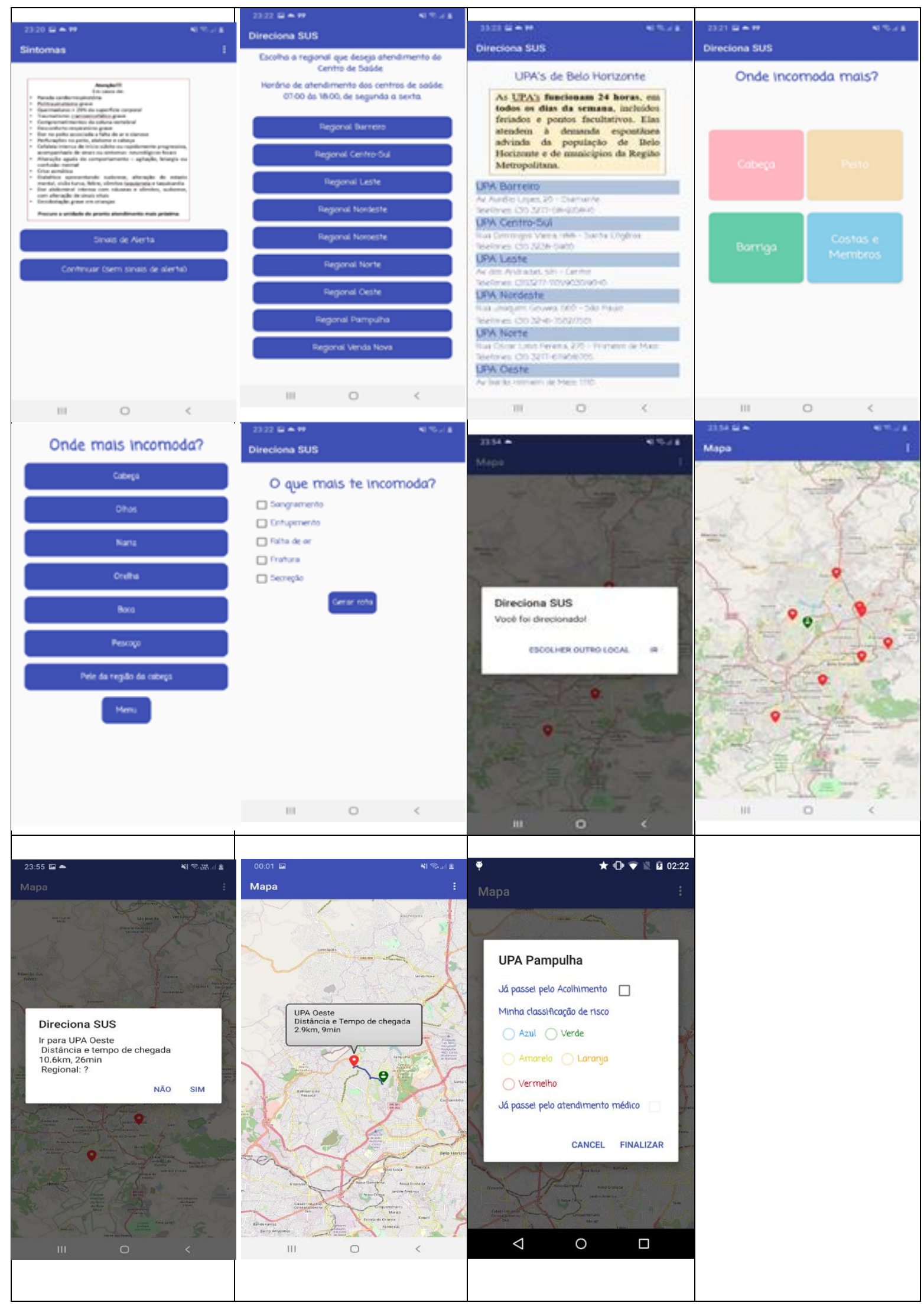

Source: (Authors) 